\title{
ANTEIL DES INTERNATIONALEN HANDELS AM BIP
}

In der zunehmend globalisierten Welt von heute sind Ausfuhren und Einfuhren Schlüsselaggregate für die Analyse der Wirtschaftslage eines Landes. Wann immer sich die Wirtschaftstätigkeit in einem Land verlangsamt oder beschleunigt, kann sich das auf alle anderen Volkswirtschaften auswirken.

\section{Definition}

Die Ausfuhren von Waren und Dienstleistungen umfassen den Verkauf, den Tausch, die Schenkung oder Übertragung von Waren und Dienstleistungen (die in der Produktionsabgrenzung des BIP liegen) von Gebietsansässigen an Gebietsfremde. Analog dazu handelt es sich bei den Einfuhren um dieselben Transaktionen von Gebietsfremden an Gebietsansässige.

Nicht alle Waren müssen physisch in ein Land verbracht werden, um als Export oder Import verbucht zu werden. Verkehrsausrüstungen, von Gebietsansässigen in internationalen Gewässern produzierte Waren, die direkt an Gebietsfremde verkauft werden, und in Schiffen oder Flugzeugen konsumierte Nahrungsmittel sind nur einige Beispiele für Waren, die als Ausfuhren oder Einfuhren verbucht werden können, ohne physisch die Grenze zu überqueren.

Desgleichen handelt es sich nicht bei allen Waren, die in ein Land verbracht werden, unbedingt um Einfuhren oder Ausfuhren. Verkehrsausrüstungen oder zur geringfügigen Bearbeitung ins Ausland verbrachte Waren (oder die in ihrem

\section{Überblick}

Vor der jüngsten Wirtschaftskrise war beim internationalen Handel mit Waren und Dienstleistungen - sowohl bei den Einfuhren als auch bei den Ausfuhren - im ganzen OECD-Raum ein stetiger Anstieg zu beobachten, wobei das OECD-Gesamtvolumen im Zeitraum 2004-2008 bei beiden Messgrößen um durchschnittlich 4-5 Prozentpunkte wuchs und die Einfuhren geringfügig schneller expandierten als die Ausfuhren. 2009 sanken im Verlauf der jüngsten Krise jedoch sowohl die Einfuhren als auch die Ausfuhren als Anteil am BIP deutlich, was den seit 2004 verzeichneten Anstieg nahezu völlig zunichte machte. Der Anteil der Ausfuhren am BIP lag 2009 mit 24,9\% erheblich niedriger als 2008 (28,0\%). Dieses Muster spiegelte sich im Anteil der Einfuhren am BIP des gesamten OECD-Raums wider, der im Zeitraum 2008-2009 durchschnittlich von 29,6\% auf 25,2\% sank. 2010 konnte beim Anteil der Einfuhren und der Ausfuhren mehr als die Hälfte der vorherigen Einbußen wiedergutgemacht werden. Diese Expansion setzte sich 2011 für nahezu alle Länder fort, für die Daten verfügbar sind. In der Mehrzahl dieser Länder liegt der Anteil der Einfuhren und der Ausfuhren nun wieder höher als vor der Krise.

Was den Saldo von Ausfuhren und Einfuhren betrifft, weisen Luxemburg, Norwegen, die Schweiz und Irland durchgehend hohe Überschüsse von über $10 \%$ des BIP auf, während die Niederlande, Schweden und Deutschland Überschüsse von über $5 \%$ verzeichnen. In den südeuropäischen Ländern (insbesondere Griechenland und Portugal), in Mexiko, im Vereinigten Königreich und in den Vereinigten Staaten ist die Handelsbilanz mit der übrigen Welt hingegen anhaltend defizitär.
Originalzustand und ursprünglichen Eigentumsstatus in ein Land gebracht werden und es so auch wieder verlassen) sind Beispiele für Waren, die die Grenze überqueren, jedoch nicht als Einfuhren oder Ausfuhren verbucht werden.

\section{Vergleichbarkeit}

Waren machen den Großteil der Ein- und Ausfuhren aus; sie sind in der Regel zufriedenstellend erfasst, was eine gute Vergleichbarkeit zwischen den einzelnen Ländern ermöglicht. Die zwischen den gesamten Wareneinfuhren und -ausfuhren auf globaler Ebene zu beobachtenden Unterschiede zeigen allerdings, dass die Frage der angewandten Messmethoden in der Praxis nicht unbedeutend ist. Die Zunahme des Internethandels hat die Messprobleme zusätzlich erhöht.

Die Vergleichbarkeit des Dienstleistungshandels wird indessen stärker durch praktische Messprobleme beeinträchtigt, selbst wenn der konzeptuelle Ansatz, wie bei den Waren, für alle OECD-Länder derselbe ist.

Bis vor kurzem handelte es sich bei den Ausfuhren und Einfuhren von Dienstleistungen hauptsächlich um Verkehrsleistungen (See-, Luftverkehr) und Versicherungsleistungen. Durch die Zunahme des Outsourcing, des Transithandels, der Bearbeitungsdienste und der Transaktionen im Bereich des geistigen Eigentums, wie Software und künstlerische Originale, haben sich jedoch die Schwierigkeiten bei der Messung des Dienstleistungshandels erhöht.

\section{Quelle}

- OECD (2012), National Accounts of OECD Countries, OECD Publishing.

\section{Weitere Informationen}

\section{Analysen}

- OECD (2012), Policy Priorities for International Trade and Jobs, OECD Publishing.

- OECD (2011), Globalisation, Comparative Advantage and the Changing Dynamics of Trade, OECD Publishing.

\section{Statistiken}

- OECD (2012), International Trade by Commodity Statistics, OECD Publishing.

- OECD (2012), Statistics on International Trade in Services, OECD Publishing.

- OECD (2011), National Accounts at a Glance, OECD Publishing.

Zur Methodik

- OECD et al. (2002), Manual on Statistics of International Trade in Services, Vereinte Nationen.

\section{Websites}

- OECD International Trade and Balance of Payments Statistics, www.oecd.org/std/its 


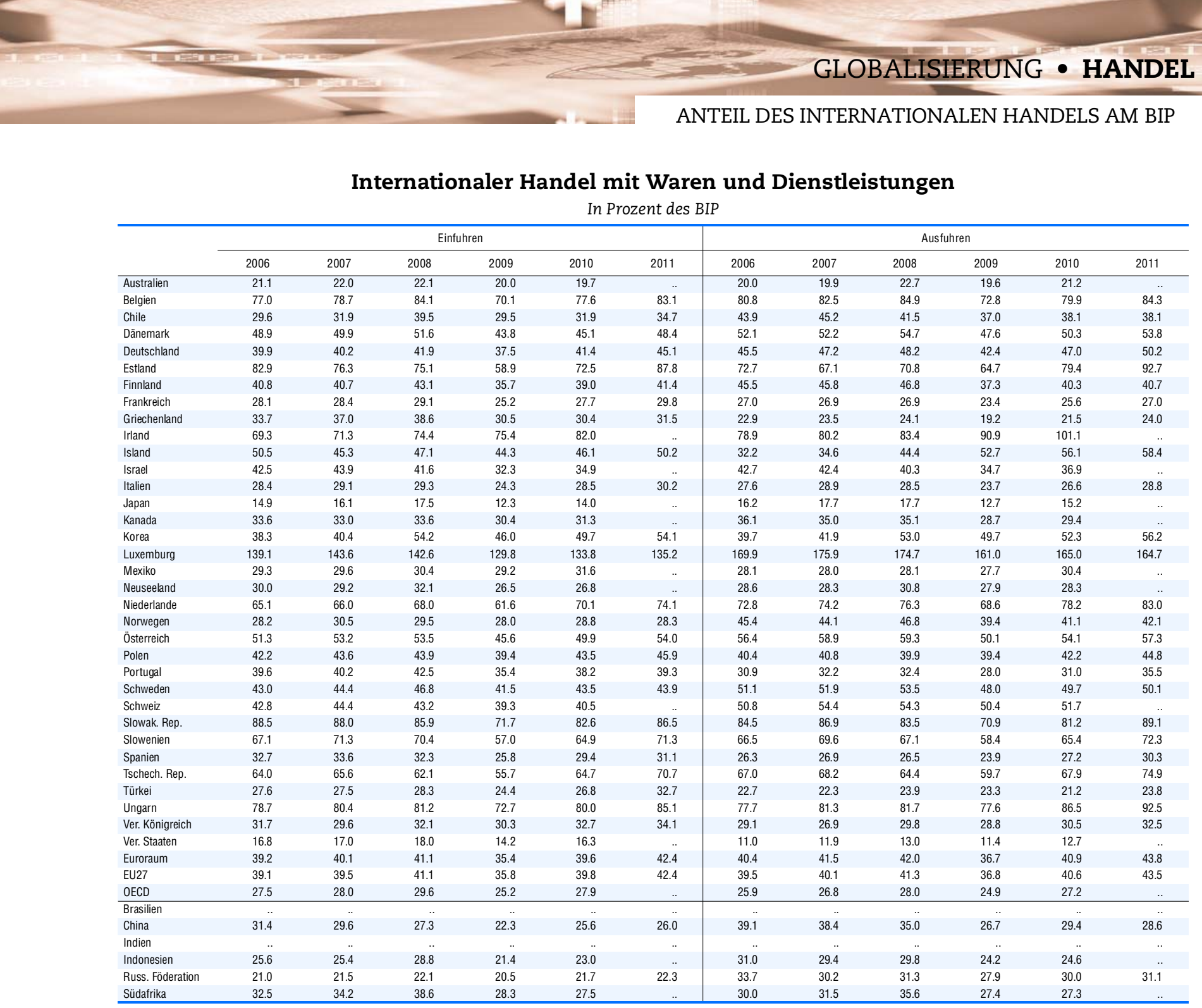

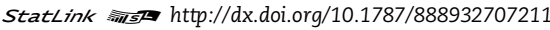

\section{Internationale Einfuhren und Ausfuhren von Waren und Dienstleistungen}

In Prozent des BIP, 2011 oder letztes verfügbares Jahr

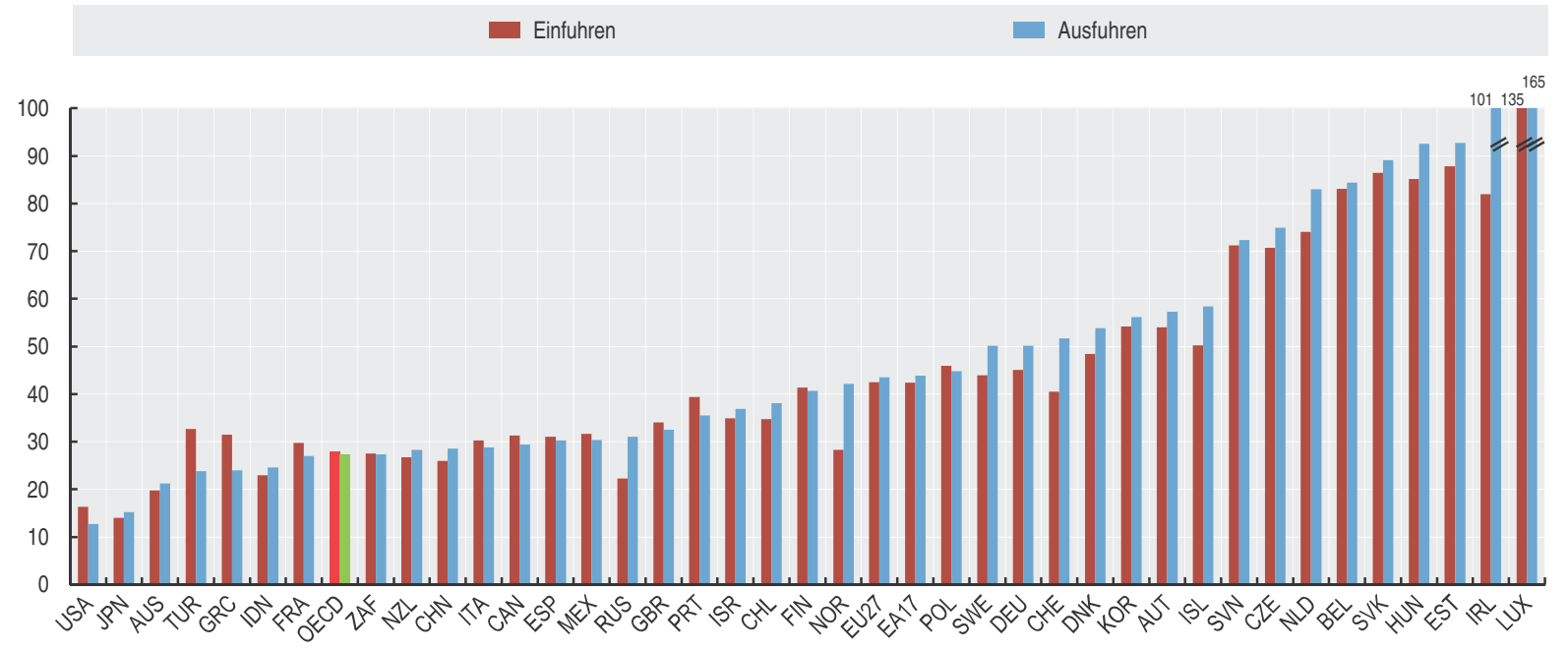




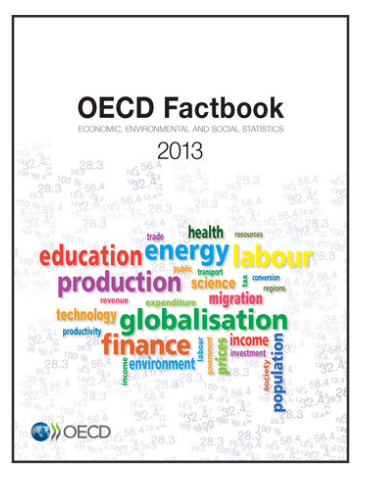

\section{From: \\ OECD Factbook 2013 \\ Economic, Environmental and Social Statistics}

Access the complete publication at:

https://doi.org/10.1787/factbook-2013-en

\section{Please cite this chapter as:}

OECD (2013), "Anteil des internationalen Handels am BIP", in OECD Factbook 2013: Economic, Environmental and Social Statistics, OECD Publishing, Paris.

DOI: https://doi.org/10.1787/factbook-2013-30-de

Das vorliegende Dokument wird unter der Verantwortung des Generalsekretärs der OECD veröffentlicht. Die darin zum Ausdruck gebrachten Meinungen und Argumente spiegeln nicht zwangsläufig die offizielle Einstellung der OECDMitgliedstaaten wider.

This document and any map included herein are without prejudice to the status of or sovereignty over any territory, to the delimitation of international frontiers and boundaries and to the name of any territory, city or area.

You can copy, download or print OECD content for your own use, and you can include excerpts from OECD publications, databases and multimedia products in your own documents, presentations, blogs, websites and teaching materials, provided that suitable acknowledgment of OECD as source and copyright owner is given. All requests for public or commercial use and translation rights should be submitted to rights@oecd.org. Requests for permission to photocopy portions of this material for public or commercial use shall be addressed directly to the Copyright Clearance Center (CCC) at info@copyright.com or the Centre français d'exploitation du droit de copie (CFC) at contact@cfcopies.com. 